\title{
Light scattering at small angles by atmospheric irregular particles: modelling and laboratory measurements
}

\author{
T. Lurton ${ }^{1}$, J.-B. Renard ${ }^{1}$, D. Vignelles ${ }^{1}$, M. Jeannot ${ }^{1}$, R. Akiki ${ }^{2}$, J.-L. Mineau ${ }^{2}$, and T. Tonnelier ${ }^{2}$ \\ ${ }^{1}$ Laboratoire de Physique et Chimie de l'Environnement et de l'Espace, CNRS UMR7328, 3A, \\ avenue de la Recherche Scientifique, 45071 Orléans, France \\ ${ }^{2}$ Environnement S. A., 111, boulevard Maximilien-Robespierre, 78300 Poissy, France \\ Correspondence to: T. Lurton (thibaut.lurton@cnrs-orleans.fr)
}

Received: 19 June 2013 - Published in Atmos. Meas. Tech. Discuss.: 21 August 2013

Revised: 13 February 2014 - Accepted: 18 February 2014 - Published: 7 April 2014

Abstract. We have investigated the behaviour of light scattering by particulates of various sizes $(0.1 \mu \mathrm{m}$ to $100 \mu \mathrm{m})$ at a small scattering angle (below $20^{\circ}$ ). It has been previously shown that, for a small angle, the scattered intensities are weakly dependent upon the particulates' composition (Renard et al., 2010). Particles found in the atmosphere exhibit roughness that leads to large discrepancies with the classical Mie solution in terms of scattered intensities in the low angular set-up. This article focuses on building an effective theoretical tool to predict the behaviour of light scattering by real particulates at a small scattering angle.

We present both the classical Mie theory and its adaptation to the case of rough particulates with a fairly simple roughness parameterisation. An experimental device was built, corresponding to the angular set-up of interest (low scattering angle and therefore low angular aperture). Measurements are presented that confirm the theoretical results with good agreement. It was found that differences between the classical Mie solution and actual measurements - especially for large particulates - can be attributed to the particulate roughness.

It was also found that, in this low angular set-up, saturation of the scattered intensities occurs for relatively small values of the roughness parameter. This confirms the low variability in the scattered intensities observed for atmospheric particulates of different kinds.

A direct interest of this study is a broadening of the dynamic range of optical counters: using a small angle of aperture for measurements allows greater dynamics in terms of particle size. Thus it allows a single device to observe a broad range of particle sizes whilst utilising the same electronics.

\section{Introduction}

The importance of measuring the concentrations and masses of aerosols in the atmosphere has been highlighted by various studies (Zemp et al., 1999; Brunekreef and Holgate, 2002), given the possible effect of their presence in ambient air upon human health. A public directive on air quality was published (European Parliament, 2008) in order to establish standards for the protection of human health. In this context, the observation of aerosols appears of great relevance, and should be made in accordance to the established norms. Particulate matter (PM) is commonly classified in size bins $\mathrm{PM}_{10}$ standing for example for particulates of aerodynamic size less than $10 \mu \mathrm{m}$. Due to the large variety in the nature and size of particulates found in the environment, and their related properties and impacts, it is of utmost importance to distinguish between those different classes.

In order to monitor aerosols, optical particle counters (OPCs) can be utilised. Their principle relies on the scattering of light by small particulates: an incident monochromatic laser beam is directed towards the particulates and scattered in space. This phenomenon is fairly well described by Mie's theory (Mie, 1908), in which the intensity of the scattered light strongly depends on the scattering angle of observation. Parameters such as the refractive index, the size of the particulate and its shape are key factors in the scattering process. In an OPC, the light flux is collected at a defined angle in order to yield information about the particulates. One can thus retrieve the size of the particulates, under certain assumptions regarding their refractive index and shape (Dick et al., 1994). Standard OPCs are able to deliver both the concentrations 
and size distributions of particulates in a continuous and realtime operating system (Rosen, 1964; Deshler et al., 2003; Donateo et al., 2006; Fischer and Koshland, 2006; McMurry, 2000; Renard et al., 2010, 2008). An example of an OPC is STAC (Stratospheric and Tropospheric Aerosols Counter). This device, dedicated to balloon flights, is operated by the LPC2E and works at a large scattering angle $\left(70^{\circ}\right)$ and under a broad field of view $\left(70^{\circ}\right)$. It is able to yield concentration profiles for particulates of diameters greater than $0.35 \mu \mathrm{m}$ (liquid) and $1 \mu \mathrm{m}$ (solid). Within an OPC, it is also possible to place detectors at several angles at the same time (Eidhammer et al., 2008), enabling cross-comparison: depending on the angle, the scattered flux is more or less sensitive to either the nature or size of the particulate. At low scattering angles, below $20^{\circ}$, the scattered light has a much stronger dependence on the particulates' size than on their nature (Renard et al., 2010). Here we make it clear that by "nature", we refer to the physical composition of the particulate studied. We do not make any assumption about the roughness, which can be of any kind: for instance, a glass bead can be relatively smooth, whereas a glass shard will be broken and fairly rough.

Because real atmospheric particulates are not strictly spherical, the classical Mie solution cannot be assumed to be wholly valid as a scattering model; the roughness of the particulates has an impact on the scattered fluxes.

The global aim of this article is to study light scattering by irregular particulates at a small angle. Throughout our article, "small angles" shall refer to angles smaller than $20^{\circ}$. Two main points should be immediately emphasised: first, given the weak dependence on the particulates' nature, this type of measurement yields first and foremost information about the size of the particulates, which constitutes the practical application of such measurements; secondly, a low scattering angle naturally entails a narrow field of view. In a first part of this paper, we present a theoretical model for the scattering by irregular particulates. Then, we confirm the model by measurements. Some discussion follows, and conclusions and perspectives are drawn for practical aerosol monitoring applications.

\section{Mie scattering for regular and irregular particulates}

\subsection{Classical Mie solution}

The complete Mie scattering solution was introduced by Gustav Mie in 1908 (Mie, 1908) and later formalised in a more concise way through the use of Bessel functions (van de Hulst, 1957). It is, on the one hand, rather long to detail comprehensively and, on the other hand, well known in its basic aspects. Therefore, we will only point out the main facts of interest for this study.
For a unit-amplitude incident light of wave number $k$ over a spherical particle, the scattered far field $\mathbf{E}_{\mathrm{s}}$ expressed in spherical coordinates $r, \theta$ and $\phi$ can be expressed as (van de Hulst, 1957)

$$
\begin{aligned}
& E_{\mathrm{s} \theta}(r, \theta, \phi)=-\frac{e^{-i k r}}{i k r} \cos \phi S_{2}(\theta) \\
& E_{\mathrm{s} \phi}(r, \theta, \phi)=\frac{e^{-i k r}}{i k r} \sin \phi S_{1}(\theta) .
\end{aligned}
$$

The $E_{\mathrm{sr}}$ radial component is assumed to be null.

The expressions of $S_{1}(\theta)$ and $S_{2}(\theta)$ are the following:

$$
\begin{aligned}
& S_{1}(\theta)=\sum_{n=1}^{+\infty} \frac{2 n+1}{n(n+1)}\left[a_{n} \pi_{n}(\cos \theta)+b_{n} \tau_{n}(\cos \theta)\right] \\
& S_{2}(\theta)=\sum_{n=1}^{+\infty} \frac{2 n+1}{n(n+1)}\left[b_{n} \pi_{n}(\cos \theta)+a_{n} \tau_{n}(\cos \theta)\right],
\end{aligned}
$$

where the $a_{n}$ and $b_{n}$ series are the usual Mie coefficients, and the $\pi_{n}$ and $\tau_{n}$ functions are angular polynomials, the expressions of which are detailed in van de Hulst (1957).

One shall note that Eqs. (3) and (4) depict an infinite series of terms, whose computation can of course not be carried out in its entirety. In order to compute values of $S_{1}$ and $S_{2}$ in simulations, one has to truncate the sum. A maximal running order is thus defined:

$N_{\text {max }}=x+4.3 x^{\frac{1}{3}}+1$,

where $x$ is the classical Mie particle parameter, $x=\frac{2 \pi}{\lambda} a$ with $a$ the radius of the particle. The sums carried out up to this order yield a maximal error of $10^{-8}$ upon the results. This formula finds justification on both empirical (Wiscombe, 1980; Mackowski et al., 1990) and theoretical grounds (Cachorro and Salcedo, 1991).

To yield the scattered light intensity at angles $\theta$ and $\phi$ and at a distance $r$, one simply sums up the squared expressions given in Eq. (1):

$$
I_{\mathrm{S}}(r, \theta, \phi)=\frac{I_{0}}{k^{2} r^{2}}\left(\left|S_{1}(\theta)\right|^{2} \sin ^{2} \phi+\left|S_{2}(\theta)\right|^{2} \cos ^{2} \phi\right) .
$$

As our study deals with small angles, we can consider that $\left|S_{1}(\theta)\right|^{2}$ and $\left|S_{2}(\theta)\right|^{2}$ do not differ greatly. Therefore, we can write the following:

$$
I_{\mathrm{S}}(r, \theta) \approx \frac{I_{0}}{2 k^{2} r^{2}}\left(\left|S_{1}(\theta)\right|^{2}+\left|S_{2}(\theta)\right|^{2}\right) .
$$

\subsection{Scattering by irregular particulates}

Methods do exist to thoroughly model light scattering by irregular particulates (Penttilä et al., 2003; Muñoz et al., 2007; Muinonen et al., 1996; Peltoniemi et al., 1989), but it is necessary to have an a priori knowledge of the shape and nature of the particulates studied. Conversely, our study uses 
all types of particulates, which is a hindrance to such traditional techniques. Moreover, we shall underline later that, for our measurements, we use averaged scattered intensities of one type and size of particulate; therefore, a statistical approach is sufficient. We propose using a simplified method to simulate light scattering by irregular particulates. The simulation scheme we use is sufficient to show that the particulate roughness has an effect upon the scattered fluxes, for a small scattering angle, a small angular aperture and for large particulate sizes. It will be shown that, as long as some moderate roughness is assumed over the particulates studied, and for the specific low angular measurement configuration, the nature of the particulate ultimately has no observable impact on the fluxes measured; the size of the particulate is then the only parameter to affect the scattered intensity.

We rely on Drossart's works (Drossart, 1990), on the one hand, and a classical diffraction approach on the other hand. In the article by Drossart (1990), a model is described to derive a statistical expression of scattered intensities for irregular particulates. The diffracted part of the signal is treated in our study through a Monte Carlo iteration process, and is used in the forward diffraction approximation realm.

We remind the reader that scattering is composed of a diffraction part and a reflection/refraction part. The derivation of this separation can for instance be found in van de Hulst (1957).

\subsubsection{Scattering by irregular particulates}

In Drossart (1990), the intensity scattered by a rough particle is investigated, and a statistical model is derived to account for irregularities of the particle shape in the scattered intensity. The method is based on using a weighting between the classical Mie solution and incoherent scattered intensities due to the random aspect of the phases of the scattered signals.

In order to lighten the notation and to concentrate on the points of interest, we remove the distance factor from the equations. They were nonetheless taken into consideration when using the model to perform our simulations.

We have thus on the one hand the coherent scattered intensities, corresponding to the classical Mie solution:

$I_{(1,2)}^{0}(\theta)=\left|S_{(1,2)}(\theta)\right|^{2}$,

and on the other hand incoherent scattered intensities, due to the surface roughness of the particle and the random phase distribution induced:

$$
\begin{aligned}
& I_{1}^{\infty}(\theta)=\sum_{n=1}^{+\infty}\left(\frac{2 n+1}{n(n+1)}\right)^{2}\left|a_{n} \pi_{n}(\cos \theta)+b_{n} \tau_{n}(\cos \theta)\right|^{2} \\
& I_{2}^{\infty}(\theta)=\sum_{n=1}^{+\infty}\left(\frac{2 n+1}{n(n+1)}\right)^{2}\left|b_{n} \pi_{n}(\cos \theta)+a_{n} \tau_{n}(\cos \theta)\right|^{2} .
\end{aligned}
$$

In Drossart (1990), the weighting of the two kinds of functions is made using a factor $f$ to yield the total scattered intensities:

$I_{(1,2)}(\theta)=f I_{(1,2)}^{0}(\theta)+(1-f) I_{(1,2)}^{\infty}(\theta)$,

where $f=\mathrm{e}^{-\sigma^{2}}$ accounts for the partial coherence of the signal. $\sigma$ is the standard deviation of the phases, defined as $\sigma=\rho x=\rho \frac{2 \pi a}{\lambda}$, with $a$ the radius of the particle, and $\rho$ is a roughness parameter. $\rho$ is defined as a scaling factor over the size discrepancies: for a given particle of mean size $\bar{x}$, a rough surface entails a possible measurement $x$ of its size through the use of statistical formula of expression

$x=\bar{x}(1+\rho \delta)$,

where $\delta$ is a statistical distribution of standard deviation 1 (Drossart, 1990).

Physically speaking, the weighting of the two types of waves can be explained as follows: the classical Mie terms are coherent solutions of the scattering by a sphere, where all the different spherical harmonics interfere. Conversely, the other terms are summed as incoherent waves. The weighting between both types of waves accounts for a coefficient of partial coherence (Drossart, 1990).

We retain this approach in the present study.

\subsubsection{Diffraction by an irregular particle}

As for the calculation of the diffracted fields by an irregular particle, we used a Monte Carlo algorithm. The particulate is considered to be of size $x$, a variable which is assigned a random value within the distribution described by Eq. (12). The corresponding diffracted field is simply calculated assuming a spherical shape of size $x$, at an angle $\theta$ :

$S_{1}^{\mathrm{d}}=S_{2}^{\mathrm{d}}=x^{2} \frac{J_{1}(x \sin \theta)}{x \sin \theta}$.

The derivation of this result can be found for instance in van de Hulst (1957), and represents no more than the wellknown fact that the Fraunhofer diffraction pattern of a circular aperture (here, the cross section of the particle) is expressed through the use of a Bessel function of first order.

By repeating this calculation a large number of times, one yields a mean diffracted intensity $I^{\mathrm{d}}(\theta)$ which accounts for the various projected sizes of the irregular particle within the light beam. This statistical approach is motivated by the fact that the experimental data are averaged over multiple singleparticle measurements.

\subsection{Synthesis for irregular particulates}

Following Drossart (1990), we operate a weighting process to account for the forward direction coherence: the diffraction approximation is met within a solid angle $\theta=\frac{\sigma \beta_{1}}{0.1 x}$, where $\beta_{1} \approx 3.83$ is the first zero of the $J_{1}$ Bessel function. 


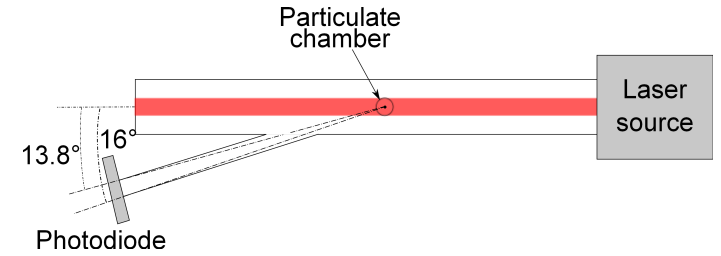

Fig. 1. Basic schematics of the experimental device used.

Within this angle, only the diffracted parts of our calculation are retained.

Such a theoretical approach must be validated by experimental data. In the following section, we detail an experimental device used for that purpose.

\section{Experimental studies}

\subsection{Description of the experimental device}

The device used to gather the experimental data in this study basically consists of a particulate chamber illuminated by a laser beam $(\lambda=635 \mathrm{~nm})$ having a power of $25 \mathrm{~mW}$, and an optical canal at the end of which a photodiode gathers the scattered light. This canal is set up at a scattering angle of around $15^{\circ}$, with the angular field of view of the photodiode lying between $13.8^{\circ}$ and $16^{\circ}$. The lower angular limit is defined to avoid direct view of the laser beam in the field of view. The upper limit marks the angular domain when the scattering light becomes sensitive to the particulates' nature (Renard et al., 2010). No lenses were used to gather the scattered light; scattered photons travel directly to the photodiode, which presents a diameter larger than the optical canal.

We use a laser beam which is linearly partially polarised. As long as the detector is not polarised, it yields the total scattered flux. Though roughness could alter the polarisation of the beam, working at a small angle of scattering makes the polarisation and depolarisation phenomena negligible for irregular grains.

The particulate chamber is such that one single particle passes through the light beam at a time. Basic schematics of the device can be found in Fig. 1. The experimental device can provide counting in 20 classes. The classes are defined by thresholds (in $\mathrm{mV}$ ) which are chosen during measurement session.

The present article focuses mainly on irregular particulates. As a consequence, the uncertainty over measured sizes is naturally high: the "size" of the particulate is all a matter of definition, be it projected or equivalent diameter. Thus, a degree of uncertainty over the measured particle size is, as a matter of fact and to our knowledge, inherent to each and every OPC. Here, our technique of measurement considers equivalent diameter.

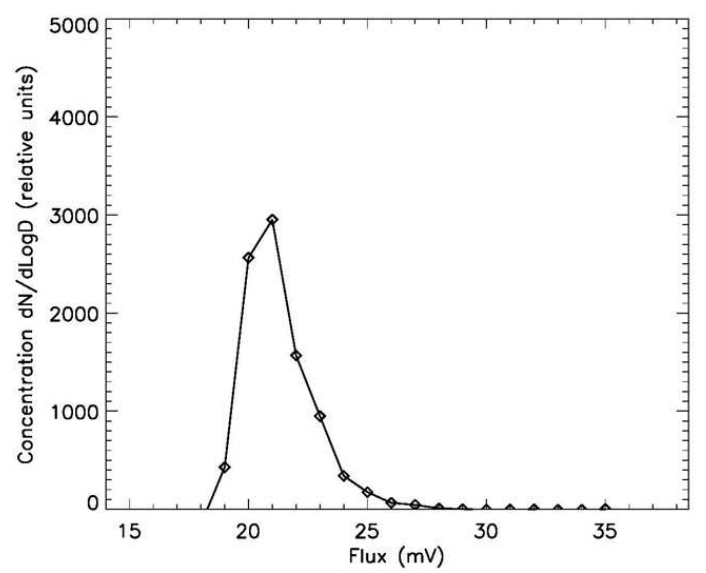

Fig. 2. Measurements for latex beads of $400 \mathrm{~nm}$. The maximum occurs at around $21 \mathrm{mV}$.

\subsection{Samples used for experiments}

Our study is based on a set of data gathered over a large variety of particulates: latex beads (in the $0.1-2 \mu \mathrm{m}$ range), glass beads (in the 5-20 $\mu \mathrm{m}$ range), and sands, salts, carbons, corundum, and volcanic ashes (in the 5-100 $\mu \mathrm{m}$ range). Latex beads are likely to be perfectly spherical, and are supposed to reproduce accurately the Mie solution, whereas the rest of the samples represent a variety of irregular particulates. (Note that we also consider glass beads as semi-irregular, since they can easily be misshapen or broken.)

For the measurement of latex beads, which are provided in liquid medium, we have used the aerosol generator TOPAS ATM 220. Latex beads are diluted in "pure" water. The aerosols travel in a chamber containing highly hydrophilic silica gel beads, to remove the droplets and water around the beads. Nevertheless, submicronic solid and liquid residuals remain in the air that is injected in the optical counting system, which could bias the calibration. Also, even if a cleaning procedure of the system is performed after each session of measurements, some latex beads used for previous measurements could remain in the aerosol generator system. We use a differential method for the measurement analysis. First, a reference measurement is carried out using only pure water. Then, this measurement is subtracted from the latex bead measurements. If the contamination is too strong, we can also subtract the measurements obtained previously with beads having smaller diameters. The highest value of the counting (or concentrations) provides the threshold in $\mathrm{mV}$ for a given size of beads. Figs. 2 and 3 present examples of measurements after applying this procedure.

The calibration for irregular particulates larger than $5 \mu \mathrm{m}$ is also conducted by a differential method, to remove the contribution of the smaller particulates. For all samples, particulates with different diameters are obtained by using sifters (meshes of $5 \mu \mathrm{m}, 10 \mu \mathrm{m}, 25 \mu \mathrm{m}$ and $32 \mu \mathrm{m}$ in diameter). 


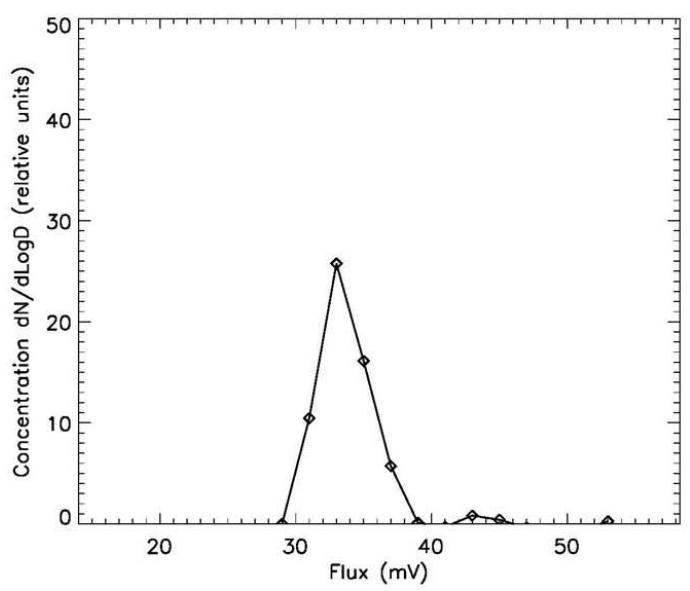

Fig. 3. Measurements for latex beads of $900 \mathrm{~nm}$. The maximum occurs at around $33 \mathrm{mV}$.

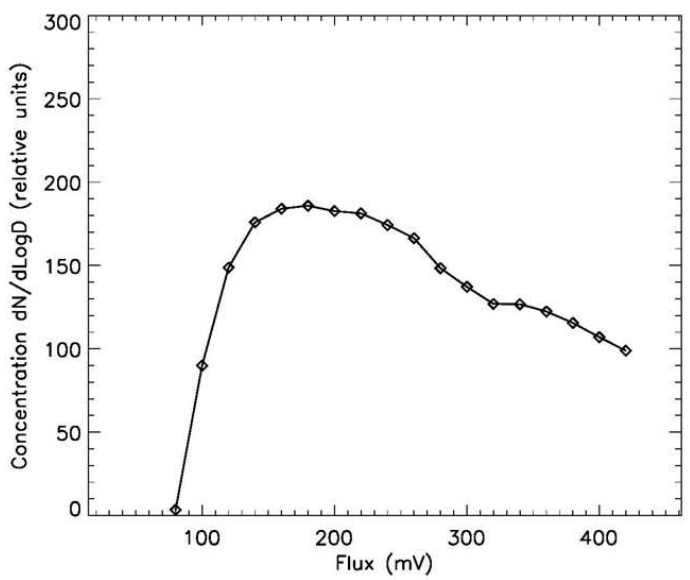

Fig. 4. Measurements for volcanic ashes through a sifter of $25 \mu \mathrm{m}$ (after subtracting the measurement through a $10 \mu \mathrm{m}$ sifter). The maxima occur at around $200 \mathrm{mV}$ and $350 \mathrm{mV}$ for diameters of $25 \mu \mathrm{m}$ and $35 \mu \mathrm{m}$, respectively.

Concentrations at a given size are retrieved from the measurements by subtracting those at the size below. Figure 4 presents the result for volcanic ashes through a $25 \mu \mathrm{m}$ sifter. Two maxima of concentration are present. The first one is assumed to correspond to concentrations of particulates having a diameter $D$ smaller than the sifter mesh. The second one is assumed to represent particulates having a diameter smaller than the diagonal $D \sqrt{2}$ of the mesh. Thus, it is possible to retrieve two calibration values of scattered intensities (flux) versus size for each sifter by considering the value of the flux at the end of the maxima.

For glass beads, we considered the size distribution provided by the manufacturer instead of the mesh size (the sifters are only used to remove pieces of broken beads and aggregates). For particulates around $100 \mu \mathrm{m}$ diameter (silicon carbide), we considered the size distribution provided by the

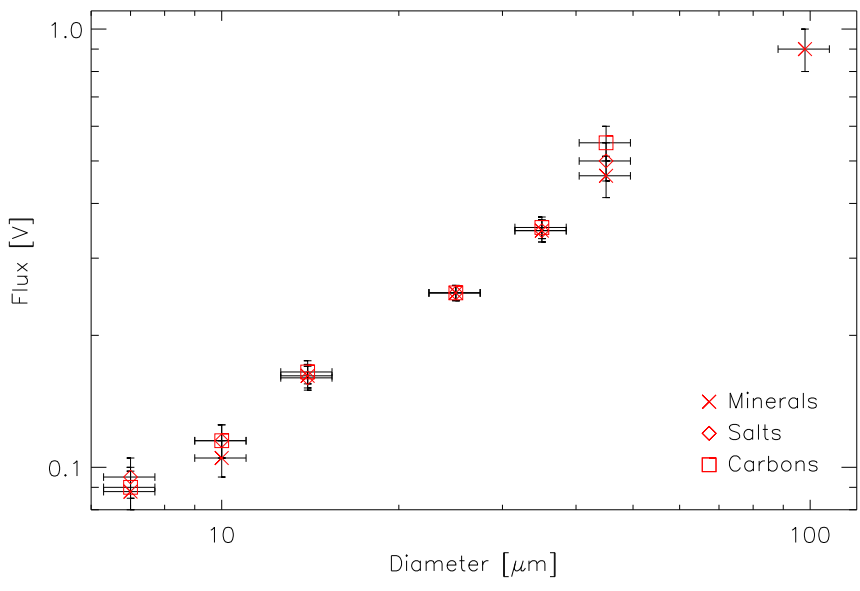

Fig. 5. Scattered intensities for different types of particulates. Abscissa is diameter of the particulates, and ordinate is the scattered flux in arbitrary units (mV), proportional to the actual light intensity.

manufacturers. Thus, the relation between flux and size was derived by considering the position of the maximum concentration distribution.

Results for particulates of different nature (minerals, carbons, salts) are averaged together to study the possible dependence of scattered flux. Figure 5 shows the scattered intensities for several particulate sizes and for different particulate natures. As expected, the dispersion is very low, proving the independence of the scattered intensity with regard to the particulates' nature at such low scattering angles.

\subsection{Estimation of the experimental roughness parameters}

This paragraph aims to establish the link between the theoretical model previously presented and the real set of data gathered through experiments. In order to quantify the roughness parameters of solid particulates, values were calculated from direct analysis of microscope images of the particulates. For each cross section delineated on a picture, the centre of gravity was determined, and the statistical distribution of the distances between points at the edge and the centre was calculated. An average over the distribution gives a mean radius, and a simple standard deviation calculation yields the roughness parameter $\rho$ (see Eq. 12). The operation was reiterated over a large number of particulates for each sample. Table 1 displays the results calculated.

\subsection{Numerical simulation results and comparison with measurements}

Figure 8 displays the results of a series of simulations performed using the method described in the previous paragraphs. The geometry used was chosen to fit that of the experimental device presented: the light scattered by the 


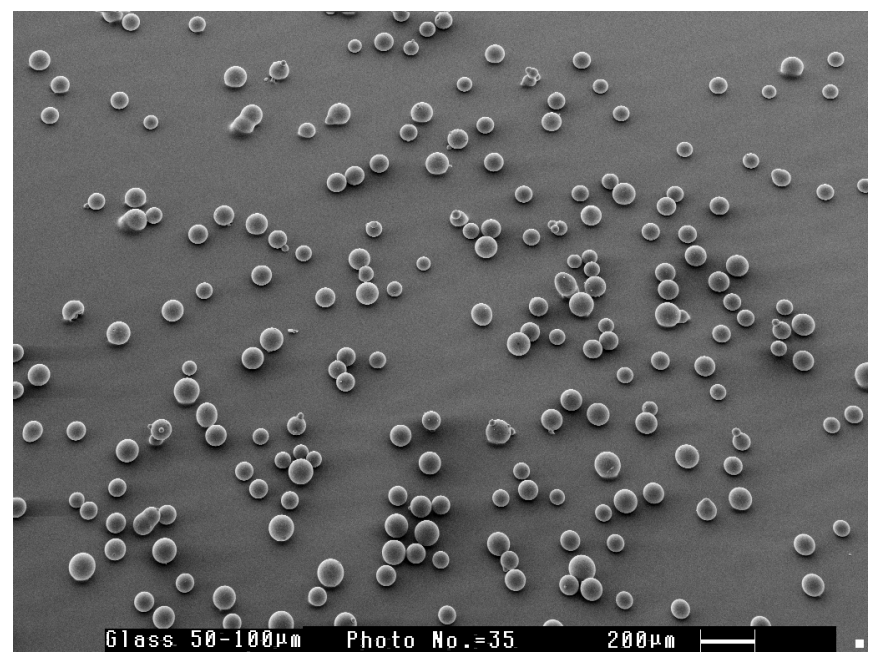

Fig. 6. Picture of glass beads used for the experiments (source: PROGRA2 database; pro, 2013).

particulates $(\lambda=635 \mathrm{~nm})$ is received under an angular aperture of $2.2^{\circ}$, at a low central scattering angle $\left(15^{\circ}\right)$. The simulation results are directly compared to the measured values.

The types of particulates used for the measurements are twofold: on the one hand, latex beads of size less than $2 \mu \mathrm{m}$, which can be considered smooth, thus are expected to match the Mie theory and, on the other hand, irregular particulates of size greater than $2 \mu \mathrm{m}$, for which no particular smoothness can be assumed. Largely overlapping sets of data (i.e. both rough and smooth particulates of the same size ranges, in particular over large sizes) are to our knowledge not easily achievable. The ATM aerosols generator cannot work for beads greater than $2 \mu \mathrm{m}$. Above that limit, powders can be generated with other kinds of generator and carried to the aerosol counting device through air flow, but they present a risk of possibly being agglomerated. Besides, it is difficult to maintain the largest particulates suspended in air without any undesired orientation effects (Daugeron et al., 2006).

For each size of rough particle, given the weak dispersion already demonstrated, a single flux value was calculated, corresponding to the average scattered intensity of the whole variety of rough particulates studied. Error bars are presented in both dimensions.

For the simulations, particulates of refractive index $m=$ 1.59 were assumed as a starting point. This value corresponds to the precise index of the regular latex beads used in the lower size range of our measurements. Firstly, the theoretical Mie scattered fluxes were calculated, upon the angular interval $\left[13.8^{\circ} ; 16^{\circ}\right]$ which is that captured by the photodiode. A $18 \mathrm{mV}$ offset was identified and added to the scattered intensities, corresponding to the electronic offset of the instrument.

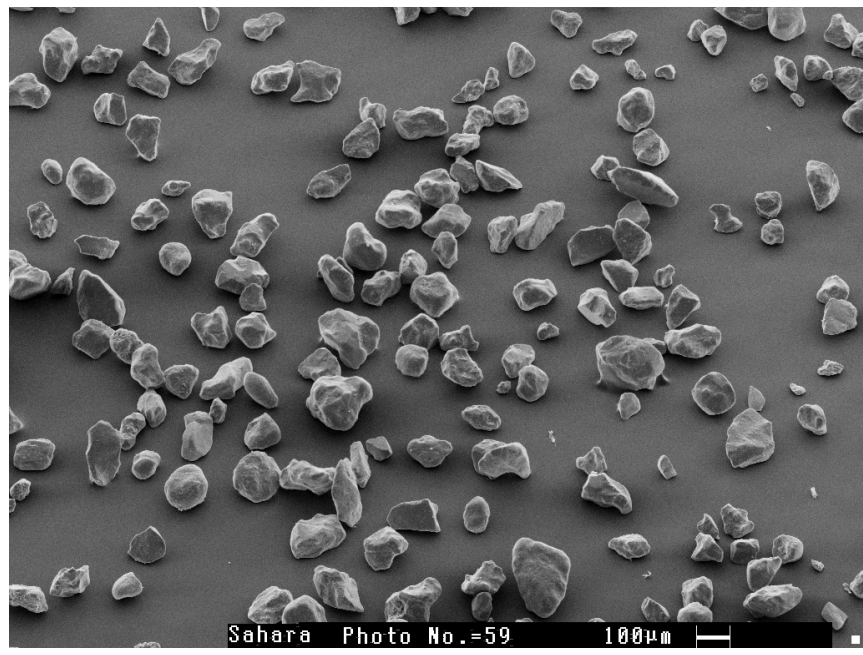

Fig. 7. Picture of sand grains used for the experiments (source: PROGRA2 database; pro, 2013).

Table 1. Estimated roughness parameters for some of the samples studied.

\begin{tabular}{lrl}
\hline Sample & Mean diameter & Estimated $\rho$ \\
\hline Glass beads & $100 \mu \mathrm{m}$ & $6.24 \times 10^{-3}$ \\
Sand & $85 \mu \mathrm{m}$ & 0.136 \\
Corundum & $120 \mu \mathrm{m}$ & 0.151 \\
Volcanic ashes & $20 \mu \mathrm{m}$ & 0.173 \\
Salt & $310 \mu \mathrm{m}$ & 0.247 \\
\hline
\end{tabular}

Following this first step, several different roughness parameters were introduced in the model, ranging from $10^{-3}$ to 0.01 , in order to simulate the detection of real, rough particulates. As one can identify in Fig. 8, the larger the roughness parameter, the more severe the discrepancy induced with respect to the Mie theory. This affects both the overall scattered intensity - which lowers as the roughness parameter grows - and the trend in the slope of the intensity vs. diameter. Note that the simulated intensities in the roughness model are only displayed for particle sizes greater than twice the wavelength used; as a matter of fact, the roughness of the particulates cannot be seen under that limit. Therefore, the Drossart model, though consistent with the Mie theory under the 2- $\lambda$ limit, does not make real physical sense for those particle sizes. Note that in Fig. 8 a slight smoothing of the curves was performed, with a sliding window of span $1 / 50$ of the total size range.

As shown in Fig. 8, there is a perfect agreement between model and measurements for the smallest sizes. On the other hand, the scattered flux for irregular solid particulates is lower than the classical Mie solution would predict for regular particles of the same size; the irregular particles measurements are nonetheless reproduced by the roughness model. 


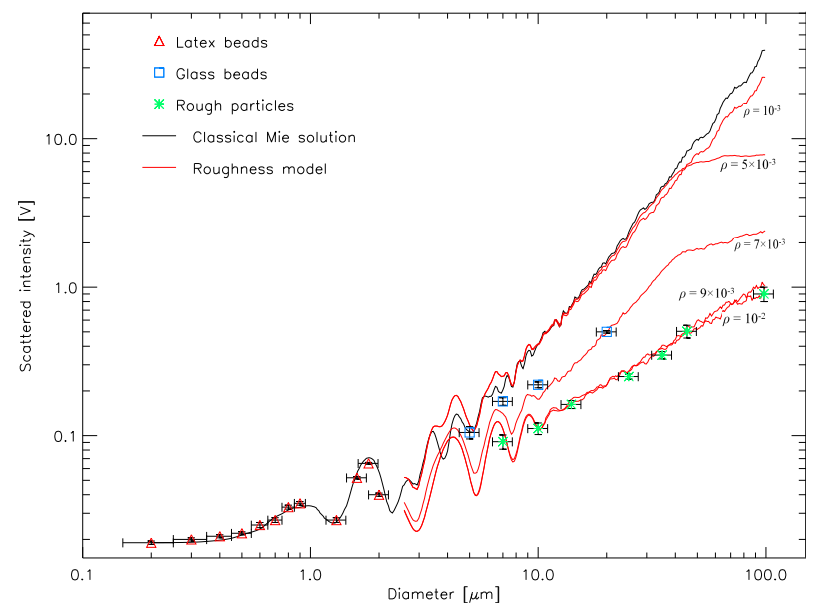

Fig. 8. Comparison of measurements for different kinds of particulates with both the classical Mie solution (plain black line) and the roughness scattering model, for different roughness parameters (plain red lines). The abscissa is the diameter of the particles studied, and the ordinate is the scattered light flux in an arbitrary unit $(\mathrm{mV})$, directly proportional to the actual light intensity. Note that the two curves for $\rho=9 \times 10^{-3}$ and $\rho=10^{-2}$ are almost identical, due to the saturation phenomenon explained in the text.

Figure 8 also shows that a lower saturation limit is quickly reached when increasing the roughness parameter. By this saturation phenomenon, we mean that the scattered intensities ultimately reach a plateau once a certain degree of roughness is exceeded. We can estimate that the saturation regime in our precise geometrical set-up is encountered for $\rho \geq 0.01$, regardless of the refractive index parameter. As a matter of fact, this saturation regime corresponds to a dominant diffraction effect of the rough particulates; as the refractive index does not play any role in the diffraction process, it is understood that the scattering flux data of all types of particulates shall collapse on the same curve. The abovementioned saturating roughness parameter value is relatively low and is very easily met by any kind of irregular particle found in the atmosphere (see Table 1). The fact that the diffraction part is the main contribution to the scattering process for large and rough particles has already been utilised by studies such as Ulanowski et al. (2012). In these studies, the size of complex-shaped particulates is retrieved through the estimation of diffraction patterns.

Glass beads present an intermediate behaviour, plotted between the standard Mie solution and the roughness model. This is wholly coherent with the expected surface state of glass beads: they are supposed to be fairly smooth, yet possibly exhibiting a broken surface, some agglomeration or being misshapen (this can be noticed on Fig. 6). Their roughness parameter should thus be low, though not perfectly null (see Table 1 for a numerical estimation of the roughness parameter of glass beads). Note for completeness that the refractive index of the glass beads used $(m=1.56)$ is close to the 1.59 value assumed for the simulations. As a matter of fact, running the simulation taking into account this slight parameter difference does not yield major changes: the Mie oscillations undergo a minor shift, but the intensities for middlesized particulates remain unchanged.

We have also ascertained, through numerical simulation, that the larger the angular aperture, the less marked the flux discrepancy effect, or put in different terms - the larger the aperture, the higher the roughness parameter needed to lead to saturation. Because we are restraining ourselves to a narrow field of view for experimental studies, these results shall not be presented thoroughly here, but could constitute some interesting matter for further studies.

\section{Discussion}

The model calculations were computed assuming a refractive index close to that of the latex beads used for the experiments. We could as well have assumed a different index (e.g. that of a carbon particulate). This would have resulted in a lower curve for the regular Mie solution; however, we would also have encountered a lower saturation phenomenon when increasing the roughness parameter.

Also, we have investigated - though not presented here the effect of a broadening of the field of view. It was ascertained through simulations that the larger the field of view, the less sensitive the effect of the particle roughness. This confirms the fact that a narrow field of view is interesting in order to yield the saturation process, and thus to lower the scattered intensities for large particulate sizes.

A direct interest of this study is that, due to the small scattering angle and angular aperture, the decrease in the intensities for large sizes of particulates naturally enables a broader dynamic range over the measurements. Thus, it becomes possible to monitor a wider range of particle sizes whilst relying on a single photodiode and electronics device. The geometry of our measurements has reduced by a factor 10 the intensity of light scattered for the largest particulates $(100 \mu \mathrm{m})$ with respect to the classical Mie theory. This opens up the possibility to detect larger particulates than using standard aerosol counters, whilst also detecting the smallest aerosols near $0.2 \mu \mathrm{m}$.

In our experimental procedure, we were required to choose a compromise between the accuracy of size determination for the smallest particulates and the dynamics of the diameter range. The accuracy is approximately $\pm 0.05 \mu \mathrm{m}$ for diameters below $1 \mu \mathrm{m}$ in our laboratory measurement device. Obviously, the accuracy of size determination for the smallest particulates can be improved by increasing the power of the light source. A $100 \mu \mathrm{m}$ particulate illuminated by light source of $100 \mathrm{~mW}$ would provide a scattered flux converted to a voltage of $4 \mathrm{~V}$, which would be close to the saturation limit of a conventional detector and electronics. In this case, the accuracy of the size determination for the submicronic aerosols would be increased by a factor of 4 . 


\section{Conclusions}

We have investigated the behaviour of light scattering by rough, natural particulates of all sizes $(0.1 \mu \mathrm{m}$ to $100 \mu \mathrm{m})$ at a small scattering angle, and monitored under a narrow field of view. Discrepancies with the classical Mie solution are found, with scattered intensities showing lower values (up to an order of magnitude for the largest particulates) than expected by the classical Mie model. These differences were attributed to the natural roughness of the particulates. A light coherence model was adapted and used to simulate the intensities measured under the experimental conditions. Good agreement was found between simulations and measured data. As for the roughness parameterisation, it was found that a relatively small roughness parameter caused the scattered intensities to collapse on a lower saturation limit corresponding solely to the diffraction part of the scattered light. This confirms the low dispersion in the scattered intensities observed for different kinds of particulates in the experiments. As this saturation limit is likely to be met even for very slightly rough particulates, the power law observed for large particle sizes can be reasonably used to estimate the sizes of any sort of real particulates found in the atmosphere.

A direct interest of this study is the conception of versatile scattering measurement devices: given the fact that the measurement dynamics are increased by the angular set-up, one can monitor a broad range of particle sizes (e.g. from $0.1 \mu \mathrm{m}$ to $200 \mu \mathrm{m}$ ) whilst relying on a single photodiode and electronics system. This will in particular be used for the new Light Optical Aerosol Counter (LOAC) which will be soon launched under all types of balloons or used at ground.

Acknowledgements. This study of light scattering was partially funded by ANR Ecotech. The authors wish to thank Claire Thaury of Environnement S.A. for some stimulating discussion upon the subject. This paper is dedicated to the memory of Jean-Luc Mineau, who passed away in 2012, and made fundamental contributions to this study.

Edited by: A. Kokhanovsky

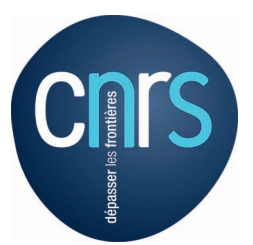

The publication of this article is financed by CNRS-INSU.

\section{References}

Brunekreef, B. and Holgate, S. T.: Air pollution and health, Lancet, 360, 1233-1242, 2002.

Cachorro, V. E. and Salcedo, L. L.: New improvements for Mie scattering calculations, J. Electromag. Waves Appl., 5, 913-926, 1991.

Daugeron, D., Renard, J.-B., Gaubicher, B., Couté, B., Hadamcik, E., Gensdarmes, F., Basso, G., and Fournier, C.: Scattering properties of sands, 1. Comparison between different techniques of measurements, Applied Optics, 45, 8331-8337, 2006.

Deshler, T., Hervig, M. E., Hofmann, D. J., Rosen, J. M., and Liley, J. B.: Thirty years of in situ stratospheric aerosol size distribution measurements from Laramie, Wyoming $\left(41^{\circ} \mathrm{N}\right)$ using balloon-borne instruments, J. Geophys. Res., 108, 4167, doi:10.1029/2002JD00251, 2003.

Dick, W. D., McMurry, P. H., and Bottiger, J. R.: Size- and composition-dependent response of the DAWN-A multiangle single-particle optical detector, Aerosol Sci. Technol., 20, 345362,1994

Donateo, A., Contini, D., and Belosi, F.: Real time measurements of PM2.5 concentrations and vertical turbulent fluxes using an optical detector, Atmos. Environ., 40, 1346-1360, 2006.

Drossart, P.: A Statistical Model for the Scattering by Irregular Particles, Astronomical J., 361, L29-L32, 1990.

Eidhammer, T., Montague, D. C., and Deshler, T.: Determination of index of refraction and size of supermicrometer particles from light scattering measurements at two angles, J. Geophys. Res., 113, 206-224, 2008.

European Parliament: Directive 2008/50/EC of the European Parliament and the Council of 21 May 2008 on ambient air quality and cleaner air for Europe, Official Journal, L152, 1-44, 2008.

Fischer, S. L. and Koshland, C. P.: Field performance of a nephelometer in rural kitchens: effects of high humidity excursions and correlations to gravimetric analyses, J. Exposure Sci. Environ. Epidemiol., 17, 141-150, 2006.

Mackowski, D. W., Altenkirch, R. A., and Menguc, M. P.: Internal absorption cross sections in a stratified sphere, Applied Optics, 29, 1551-1559, 1990.

McMurry, P. H.: A review of atmospheric aerosol measurements, Atmos. Environ., 34, 1959-1999, 2000.

Mie, G.: Beiträge zur Optik trüber Medien, speziell kolloidaler Metallösungen, Annalen der Physik, 3, 377-445, 1908.

Muinonen, K., Nousiainen, T., Fast, P., Lumme, K., and Peltoniemi, J. I.: Light scattering by gaussian random particles: ray optics approximation, J. Quant. Spectr. Radiat. Trans., 55, 577-601, 1996.

Muñoz, O., Volten, H., Hovenier, J. W., Nousiainen, T., Muinonen, K., Guirado, D., Moreno, F., and Waters, L. B. F. M.: Scattering matrix of large Saharan dust particles: Experiments and computations, J. Geophys. Res., 112, 215-232, 2007.

Peltoniemi, J. I., Lumme, K., Muinonen, K., and Irvine, W. M.: Scattering of light by stochastically rough particles, Applied Optics, 28, 4088-4095, 1989.

Penttilä, A., Lumme, K., Worms, J. C., Hadamcik, E., Renard, J.B., and Levasseur-Regourd, A. C.: Theoretical analysis of the particle properties and polarization measurements in the PROGRA2 experiment, J. Quant. Spectr. Radiat. Trans., 79, 10431049, 2003.

PROGRA2 website: available at: http://www.icare.univ-lille1.fr/ progra2/ (last access: 23 May 2013), 2013. 
Renard, J.-B., Brogniez, C., Berthet, G., Bourgeois, Q., Gaubicher, B., Chartier, M., Balois, J.-Y., Verwaerde, C., Auriol, F., Francois, P., Daugeron, D., and Engrand, C.: Vertical distribution of the different types of aerosols in the stratosphere: Detection of solid particles and analysis of their spatial variability, J. Geophys. Res., 113, D21303, doi:10.1029/2008JD010150, 2008.

Renard, J.-B., Thaury, C., Mineau, J.-L., and Gaubicher, B.: Smallangle light scattering by airborne particulates: Environnement S.A. continuous particulate monitor, Measure. Sci. Technol., 21, D21303, doi:10.1088/0957-0233/21/8/085901, 2010.

Rosen, J. M.: The vertical distribution of dust to 30 kilometers, J. Geophys. Res., 69, 4673-4676, 1964.

Ulanowski, Z., Hirst, E., Kaye, P. H., and Greenaway, R.: Retrieving the size of particles with rough and complex surfaces from twodimensional scattering patterns, J. Quant. Spectr. Radiat. Trans., 113, 2457-2464, 2012. van de Hulst, H. C.: Light Scattering by Small Particles, John Wiley \& Sons, Inc., New York, 1st Edn., 1957.

Wiscombe, W. J.: Improved Mie Scattering Algorithms, Applied Optics, 19, 1505-1509, 1980.

Zemp, E., Elsasser, S., Schindler, C., Kunzli, N., Perruchoud, A. P., Domenighetti, G., Medici, T., Ackermann-Liebrich, U., Leuenberger, P., Monn, C., Bolognini, G., Bongard, J.-P., Brandli, O., Karrer, W., Keller, R., Schoni, M.-H., Tschopp, J.-M., Villiger, N., and Zellweger, J.-P.: Long-term ambient air pollution and respiratory symptoms in adults (SAPALDIA study), Ame. J. Respiratory Critical Care Med., 159, 1257-1266, 1999. 